\title{
Near Magnetic Field of a Small Turbojet Engine
}

\author{
R. ANDOGA ${ }^{a, *}$ AND L. FÖZÖ ${ }^{b}$ \\ ${ }^{a}$ Dept. of Avionics, Faculty of Aeronautics, Technical University of Košice, Rampová 7, 04121 Košice, Slovakia \\ ${ }^{b}$ Dept. of Aviation Engineering, Faculty of Aeronautics, Technical University of Košice, \\ Rampová 7, 04121 Košice, Slovakia
}

\begin{abstract}
Different parameters like temperatures, pressures, speed, and vibrations are used in evaluation of technical state of turbojet engines. The article presents a pioneering idea to expand this set of parameters with measurements of distortions in magnetic field in a close vicinity of the engine to obtain more information about its state, which can be further used in its diagnostic and control systems. We believe that deviations in near magnetic field can hold valuable information, which can be used to predict malfunctions and evaluate the condition of the engine. The presented article shows pilot experimental results and gives answer to the basic hypothesis, if such measurements can be realized using a small turbojet engine, as well if there are significant changes in near magnetic field, which would warrant further research in the area.
\end{abstract}

DOI: 10.12693/APhysPolA.131.1117

PACS/topics: 41.20.-q

\section{Introduction}

Different information sources can be used in diagnostics and control of a turbo-jet engine. These sources are usually represented by measured values from sensors evaluating the thermodynamic process of the engine and its state parameters, like temperatures, pressures, speeds, vibrations, etc. [1]. After these parameters are measured, together with environmental parameters, they form a space which defines a state of the engine. Traditionally, these parameters are measured in different cuts of the engine by analogue and digital sensors, processed further by control and diagnostic systems [1, 2]. The need for improvement of control quality and safety of operation of aviation systems is ever present and this can be done by expansion of the parametric space of the engine by measurement of further physical characteristics of the engine, thus increasing our knowledge about the controlled object. More knowledge about the object brings more information, which can be used in the control process as well as diagnostics in order to detect and even forecast failures.

We present a pioneering idea of expanding this broad range of parameters by measurement of distortions in near magnetic field of a small turbojet engine. The basic hypothesis is that the manifestation of the engine's inner thermodynamic processes in near magnetic field can hold valuable information, which can be used to obtain diagnostic information and predict failures as well as be used to enhance the quality of its control. The article presents a concept of measurement of distortions in near magnetic field of the turbojet engine iSTC-21v and pilot data analysis showing distortions in magnetic field during controlled experimental runs of the engine. We expect the knowledge to be applicable also to other thermosenergetic technical systems.

*corresponding author; e-mail: rudolf . andoga@tuke.sk

\section{Near magnetic field of a turbojet engine}

A natural manifest of ferromagnetic parts of a small turbojet engine (iSTC-21v) is the change in density of earth magnetic field where the engine is placed. From the definition of Thomson about the energetic minima, we can expect that the magnetic domains will take a position with minimal magnetic energy [3, 4]. A magnetic conductor thus becomes an equipotential surface. Application of the Thomson definition shows that the increase in energy in a magnetic conductor can create local decrease in magnetic earth field energy. The total energy volume increases towards the equipotential surface and manifests itself as a magnetic density expressed as changing magnetic induction of the field $[3,4]$. The shape of this field is given by the magnetic conductive material and we designate this as near magnetic field — "magnetic aura" [5].

In order to confirm the previously defined hypothesis, measurements have been done in the laboratory of intelligent control systems of aircraft engines on the object of a small turbojet engine iSTC-21v. The measurements have been done during controlled runs of the engine, which is using our design of digital electronic control system maintaining precise experimental conditions. In accordance with the previously stated ideas we believe that changes in the near magnetic field are the result of changes in temperatures and rotational speeds of the engine.

\section{The experimental setup}

The measurements were done in the laboratory of intelligent systems using a small turbo-jet engine iSTC-21v with a proprietary control system design and real-time data acquisition system [5]. The magnetic field was measured using VEMA-04 magnetometer [6, 7]. The engine is shown in Fig. 1 together with the chosen arbitrary coordinate system. Magnetometer VEMA-04 was placed in the beginning of the system designated as " 0 " point in the figure. This represents a point below of the turbine of the engine, where we expect the changes of the near magnetic field to be the biggest. Individual channels of the 


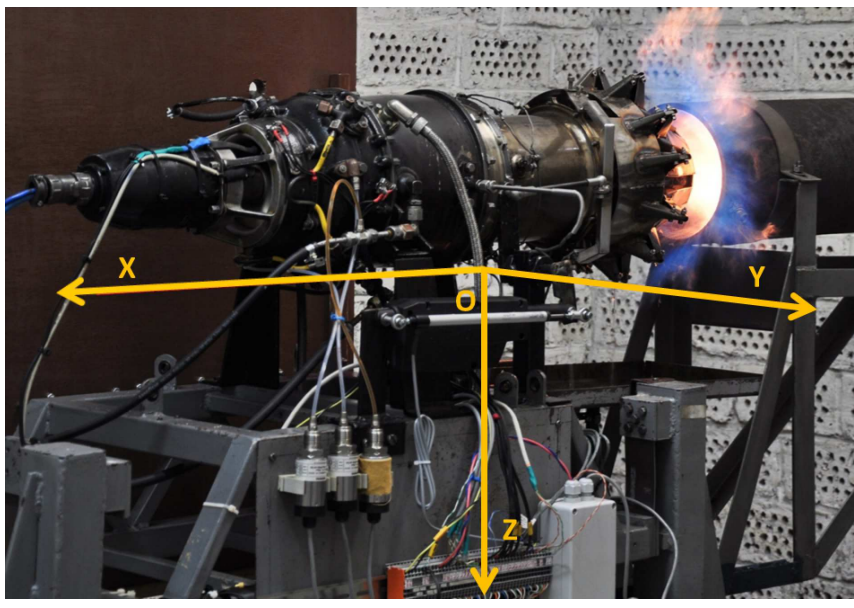

Fig. 1. Coordinate system and the experimental turbojet engine iSTC-21v.

magnetometer were placed in direction of the orthogonal axes designated as $X, Y, Z$.

Individual operational parameters of the engine are measured by a National Instruments cDAQ system converting analogue outputs of individual sensors installed on the engine to digital values with the final sampling rate of $F_{S}=10 \mathrm{~Hz}$. In this way 18 operational parameters are measured [5]. In accordance with the hypothesis of the paper in terms of magnetic field changes the temperatures and rotational speed of the engines are expected to be the most important. The time plot of a single run of the engine is shown in Fig. 2.

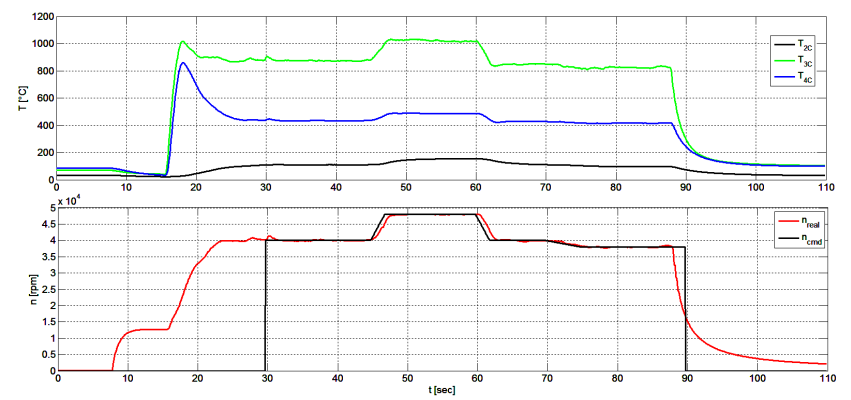

Fig. 2. A single run of the engine iSTC-21v.

The temperatures $T_{4 c}$ and $T_{3 c}$ are total temperatures in front and aft of the turbine, we can see that the engine peaks at $1000^{\circ} \mathrm{C}$ in front of the turbine and at $800^{\circ} \mathrm{C}$ aft of the turbine during startup. The speed is set to idle at 40000 RPM, after that it is commanded to accelerate to 48000 RPM. The commanded speed is shown in the figure as a black curve. This pre-programmed deterministic command signal was used for all further experiments, as we want to measure changes in the near magnetic field with changing of the rotational speed and temperatures of the engine.

The measurement of inductance of magnetic field was done using VEMA 04a magnetometer with a data sampling frequency of $F_{S}=1000 \mathrm{~Hz}$. We used three channels of the magnetometer aligned in the axes as shown in the Fig. 1.

\section{Experimental data - time domain analysis}

During a run, as shown in Fig. 2, the resulting changes in magnetic inductance as a function of time below the turbine are presented in Fig. 3.

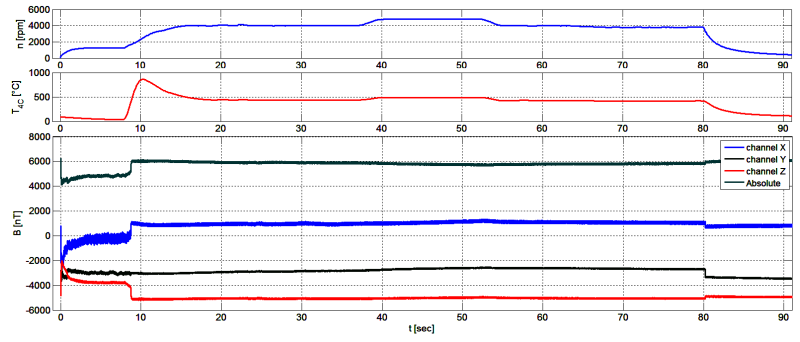

Fig. 3. Time plot of changes in magnetic induction during a single run of the engine.

The basic results of the time domain analysis of changes in magnetic induction during three consecutive runs tell us that we can positively detect the following conditions of the engine by observing sharp changes in absolute magnetic inductance computed from its three vector elements:

- Startup of its electric motor (0 seconds in the measurement in Fig. 3).

- Ignition of fuel (8th second in Fig. 3).

- Shutdown of the engine (80th second in Fig. 3).

Acceleration of the engine has also some influence in time domain, however the changes in magnetic inductance are rather small and are subject to further analysis. We are also planning to define mathematic apparatus to detect basic states of the engine, which can be used in its control system as a situational classifier [5].

\section{Experimental data - frequency domain analysis}

Because time domain changes in magnetic inductance were slow during acceleration of the engine a frequency domain analysis has been done, as changes in the frequency spectrum of the measured signal were expected to be more significant. A discrete fast Fourier transform was used [8].

The analysis was done on three consecutive runs of the engine designated as 1, 2, 3 in three areas designated as

- I - area before acceleration, RPM $=40000$,

- II - area after acceleration, RPM $=48$ 000,

- III - area after deceleration, RPM = 40000.

Each of the area was split in three two seconds blocks designated as: $a, b, c$. This is shown in Fig. 4. The results are summarized in Fig. 5. These graphs correspond to 
the individual runs of the engine. It is apparent that we can see considerable shift in dominant frequency during acceleration in each of the runs. It can be seen that the dominant frequency in area I shifts from around $167 \mathrm{~Hz}$ to the dominant frequency in the area II, which is equal to $199 \mathrm{~Hz}$ and back to $163 \mathrm{~Hz}$ after deceleration in the area III. The averaged results across engine runs and across areas are presented in Table I.

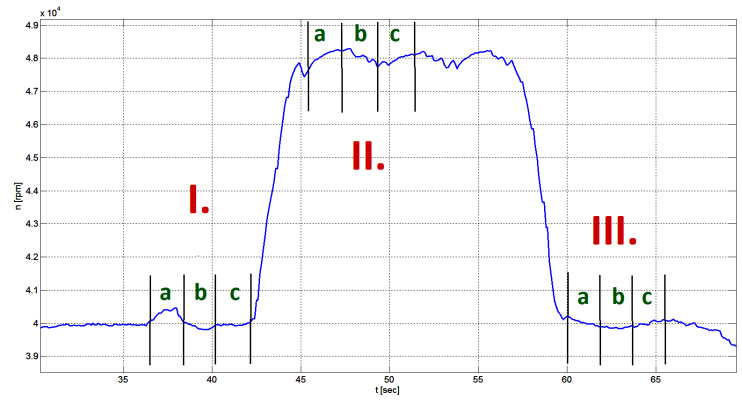

Fig. 4. Analyzed areas during a single run of the engine.
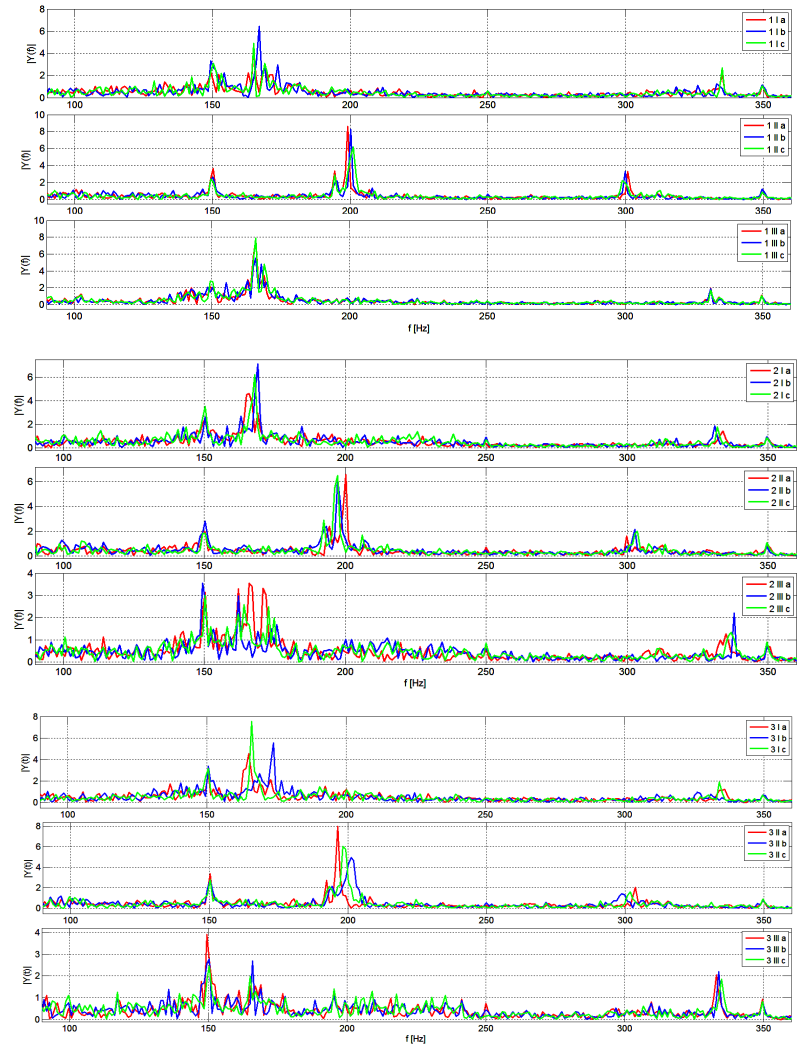

Fig. 5. Frequency analysis in three consecutive runs.

TABLE I

Frequency data analysis results.

\begin{tabular}{c|c|c|c}
\hline \hline Area & I & II & III \\
\hline average dominant frequency & $167.1 \mathrm{~Hz}$ & $199.1 \mathrm{~Hz}$ & $163.5 \mathrm{~Hz}$ \\
standard deviation & 2.82 & 1.746 & 5.63
\end{tabular}

\section{Conclusions}

We can state that the basic hypothesis of usability of measurement of distortions of magnetic field near a thermodynamic object has been confirmed. Optimal placement of the magnetometer has been defined and pilot measurements have shown that the resulting data are usable.

Time domain analysis of changes in near field magnetic induction data has shown that we can use them to classify the operational states of the engine. Further experiments are planned to find a mathematical apparatus in order to use time domain magnetic induction data for situational classification.

Frequency analysis of magnetic induction data has shown that acceleration and deceleration of the engine is changing magnetic inductance in near field around the engine. Follow up experiments will be designed in order to determine the amount of the dominant frequency shifts and to evaluate the influence of temperature and speed on the shift separately. Another set of experiments will be designed to evaluate the malfunctions and their manifestation in distortion of magnetic field.

\section{Acknowledgments}

The work presented in this paper was supported by KEGA under grant No. 014TUKE-4/2015: "Digitalization, virtualization and testing of a small turbojet engine and its elements using stands for modern applied lecturing".

\section{References}

[1] L. Jaw, C.D.J. Mattingly, Aircraft Engine Controls Design, System Analysis, and Health Monitoring, American Institute of Aeronautics and Astronautics, 2009, p. 361.

[2] G. Sanjay, NASA Glenn Research in Controls and Diagnostics for Intelligent Propulsion Systems, NASA/TM 2005-214036, 2005.

[3] J.A. Stratton, Electromagnetic Theory. The IEEE Press Series on Electromagnetic Wave Theory, J. Wiley, Hoboken (NJ) 2007, p. 616.

[4] G.B. Ezzat, J. Electrostat. 66, 561 (2008).

[5] R. Andoga, L. Madarász, L. Fözö, T. Lazar, V. Gašpar, Acta Polytechn. Hungar. 10, 81 (2013).

[6] J. Hudák, J. Blažek, D. Praslička, I. Mikita, P. Lipovský, P. Gonda, J. Electric. Eng. 61, 28 (2010).

[7] P. Lipovský, K. Draganová, M. Šmelko, T. Volčko, in: ICMT 2015 - Int. Conf. on Military Technologies 2015, art. no. 7153671 .

[8] J.W. Cooley, J.W. Tukey, Math. Computat. 19, 297 (1965). 